\title{
Factores determinantes en la implementación de manufactura esbelta utilizando la teoría del desarrollo psicosocial: caso aplicado en una empresa del sector acerero en Nuevo León (Key factors in the implementation of lean manufacturing using the theory of psychosocial development: case applied to a steel company in Nuevo León)
}

\author{
Dante Cortez, Klender Cortez, Gabriela García \& Martha Rodríguez \\ UAC, Saltillo, Coah. México, dcortez@frisa.com
}

Key words: Lean manufacturing, life cycle, psychosocial development theory, steel industry,

\begin{abstract}
This paper presents a case of the implementation of some lean manufacturing initiatives such as lean Kaizen events, improvement project, standardization, training center, "Stage Gate", ideas and suggestion, ISO 14000, ISO 17025 in a steel company in Nuevo León. This study used a diagnostic tool proposed by Hiroyuki Hirano to determine the degree of maturity of the initiative of continuous improvement, and compare against the steps suggested by the main authors in this issue. The above determine the main steps and factors of success and crises and barriers that were presented. Also, we propose an adaptation of the model proposed by Erik Erikson to organism principle, which takes into account at every stage life cycle.
\end{abstract}

Palabras clave: Ciclo de vida, industria acerera, manufactura esbelta, teoría del desarrollo psicosocial

Resumen. Este artículo presenta un estudio de caso de la implementación de algunas Iniciativas de Manufactura Esbelta como Lean- Eventos Kaizen, Proyectos de Mejora DMAIC, Equipos participativos, Estandarización, Centro de entrenamiento, Desarrollo de productos "Stage Gate", Sistema de Ideas, ISO 14000, ISO 17025 en una empresa de acero en Nuevo León. Para dicho estudio se usó la herramienta de diagnóstico propuesta por Hiroyuki Hirano para determinar el grado de madurez de la iniciativa de mejora continua, y se compara contra los pasos sugeridos por los principales autores del tema. De lo anterior se puede determinar los principales pasos y factores de éxito en la implementación así como las crisis y barreras que se presentaron. Además proponemos una adaptación del principio organísmico de las personas propuesto por Erik Erikson al Ciclo de vida de las iniciativas, donde toma en cuenta en cada estadio. 


\section{Introducción}

Uno de los principales problemas de las organizaciones en la implementación de un sistema de Manufactura Esbelta (ME) (en inglés, Lean Manufacturing) es su sostenimiento, es decir, la forma que las iniciativas de mejora continua evolucionen y perduren. Se han encontrado que las herramientas utilizadas para ello, han cambiado de nombre a través del tiempo y que la aplicación de las mismas contemplan diferentes secuencias en su implementación, ejemplo de lo anterior, son las investigaciones que se han realizado desde de las ocho disciplinas de Henry Ford en los cuarentas hasta las adaptaciones actuales de Lean Six Sigma Harry y Crawford (2005).

En esta investigación tiene como objetivo encontrar la forma de medir el nivel de madurez así como determinar los pasos y factores para la implementación de ME desde una perspectiva teórica y aplicada. Para ello, se divide la investigación en 4 partes. En la primera parte se hace una recopilación de los principales autores que estudian la implementación del ME. En segundo lugar, se analizan las iniciativas de ME en una empresa del sector acerero para encontrar los factores determinantes de una adecuada implementación. En tercer lugar, se hace una adaptación de la Teoría Psicosocial de Erikson (2005) a la implementación de ME. Por último, se presentan resultados y conclusiones.

La hipótesis de investigación que se propone es que el nivel de madurez de las iniciativas esta relacionado con los pasos propuestos de implementación. Para probar la hipótesis se utiliza el estudio de caso para la recopilación de la información y el método de regresión para probarla.

\section{Antecedentes}

Japón devastada por la segunda guerra mundial en mayo de 1949, vivía una crisis en sus industrias, baja calidad, altos costos de producción, la producción de una gran variedad de productos en cantidades pequeñas etc. Todos los conocimientos acumulados en las economías de escala y en torno a la producción de grandes volúmenes no servían para nada en este entorno. Ante esta situación Taichi Ohno jefe de producción de Toyota generó el Sistema de Producción Toyota (TSP) aportando un sistema de organización

\section{Cortez et al.}


del trabajo para lograr producir a bajos costos, volúmenes limitados de productos diferenciados (Liker, 2006).

Fuera de Toyota el TPS es muchas veces conocido como Lean, Lean Manufacturing $\mathrm{OME}$, estas expresiones se han hecho populares en dos best sellers "La máquina que cambio el mundo" Womack et al. (1991) y "Lean thinking" de Womack y Jones (1996) ambos autores han dejado claro que la base es el Sistema de Producción Toyota.

\section{Principios, la base de la cultura}

La gran mayoría de las empresas busca el enfoque de técnicas y herramientas ME, pero no debe perderse el enfoque cultural, los principios de gestión guía que rigen su filosofía, y a las personas que son las que toman decisiones y puede transformar la Organización en ME. A continuación presenta los 14 principios de Gestión del TPS, así como comportamientos esperados en cada uno de ellos, Liker (2006).

\section{Sección I: Filosofía a largo plazo}

Principio 1. Base sus decisiones de gestión en una filosofía a largo plazo, a expensas de lo que suceda con los objetivos financieros a corto plazo.

\section{Sección II: El proceso correcto producirá los resultados correctos}

Principio 2. Cree procesos en flujo continuo para hacer que los problemas salgan a la superficie.

Principio 3. Utilice sistemas pull para evitar producir en exceso.

Principio 4. Nivele la carga de trabajo (heijunka). (Trabaje como la tortuga, no como la liebre).

Principio 5. Cree una cultura de parar a fin de resolver los problemas, para lograr una buena calidad a la primera.

Principio 6. Las tareas estandarizadas son el fundamento de la mejora continua y de la autonomía del empleado.

Principio 7. Use el control visual de modo que no se oculten los problemas.

Principio 8. Use sólo tecnología fiable y absolutamente probada que dé servicio a su gente y a sus procesos.

\section{Manufactura Esbelta}


Sección III: Añada valor a la organización mediante el desarrollo de su personal y de sus socios

Principio 9. Haga crecer a líderes que comprendan perfectamente el trabajo, vivan la filosofía y la enseñen a otros.

Principio 10. Desarrolle personas y equipos excepcionales que sigan la filosofía de su empresa.

Principio 11. Respete su red extendida de socio y proveedores, desafiándoles y ayudándoles a mejorar.

Sección IV: La resolución continua de los problemas fundamentales impulsa el aprendizaje organizativo

Principio 12. Vaya a verlo por sí mismo para comprender a fondo la situación.

Principio 13. Tome decisiones por consenso lentamente, considerando concienzudamente todas las opciones; impleméntelas rápidamente.

Principio 14. Conviértase en una organización que aprende mediante la reflexión constante y la mejora continua.

\section{Iniciativas de Manufactura Esbelta en las industrias}

Cada empresa, consultor lo adapta de forma diferente tomando en cuenta la cultura, estrategia y misión de cada empresa. La variedad de descripciones usadas para definir los elementos de TPS, las interacciones entre las diversas escuelas y modelos, han creado mucha confusión en definiciones y términos para las personas que quieren implementar de acuerdo a Goyal y Deshmukh (1992), con el paso del tiempo se están estandarizando los conceptos, queda claro que no solo es un método, si no un conjunto comportamientos y hábitos para crear cultura.

A partir de Flynn et al. (1999), Wong (2009) y Arrienta (2010) se concluye que herramientas como 5'S, Eventos Kaizen, Programa de Mejora, Kanban, JIT (Just in Time), TPM (Total Productive Maintenance), Mapas de Valor, Seis Sigma son de los más usados. También se observa que el concepto de Poka yoke, Kanban, JIT, SMED-Setup siguen vigentes en la actualidad, TQC, Teoría de restricciones, Multihabilidades, ya no salen en las

\section{Cortez et al.}


encuestas actuales. En la encuesta de White 1999, en la conclusión aporta datos donde afirma que en empresas grandes es más frecuente el uso de elementos ME que en empresas pequeñas. En los diferentes estudios se hace énfasis en la importancia de la Filosofía y Cultura, esto se analizará con los elementos de un proceso de implementación y cambio organizacional.

Investigación sobre los pasos de implementaciones de iniciativas de mejora

Existen muchas propuestas de cómo implementar una iniciativa de Mejora a continuación de enumeran los 17 pasos propuestos por Robert (2005) que se presentan en la tabla 1. De estos pasos Robert (2005) identifica 6 factores comunes $(3,5,8,12,13,14)$. Adicionalmente Womack (1991) propone 4 factores: 1) venta de idea a la dirección, 2) encontrar agente de cambio, 3) crisis, y 4) valores de la empresa.

Tabla 1. Pasos para implementar ME, Robert (2005)

\begin{tabular}{|l|}
\hline 1. Crear el Comité Directivo de seguimiento \\
\hline 2. Organizar la mejora (Incluye selección de consultor externo-interno) \\
\hline 3. Definir Visión y Objetivos \\
\hline 4. Formar/Entrenar Equipo Directivo \\
\hline 5. Establecer métricos \\
\hline 6. Diagnostico situación Actual \\
\hline 7. Perfil de prioridades, (selección Herramientas, áreas piloto) \\
\hline 8. Elaborar Plan de Mejora \\
\hline 9. Constituir el Equipo implementador \\
\hline 10. Vencer la Resistencia al Cambio \\
\hline 11. Presentar el Plan al Comité Directivo \\
\hline 12 Implementar plan (Énfasis entrenamiento a los diferentes niveles) \\
\hline 13. Comprobar los Resultados \\
\hline 14. Estandarizar las mejoras \\
\hline 15. Presentar Resultados al Comité Directivo \\
\hline 16. Dar Reconocimiento \\
\hline 17. Plantear nuevas implementaciones, o mejoras \\
\hline
\end{tabular}

Manufactura Esbelta 
Modelos para evaluar la visión, niveles de implementación.

Para iniciar un proceso de cambio es necesario saber hacia donde queremos cambiar, es decir tener una visión de cómo queremos estar a 5 años en las diferentes iniciativas, esta Visión identificará el nivel de implementación o madurez de la iniciativa de Mejora continua, posteriormente se pueden fijar los objetivos que se buscan en el corto, mediano y largo plazo.

En la práctica con las empresas, sin lugar a duda varia de acuerdo a su grado de madurez en la implementación, por eso se sugiere un Modelo de Visión, para diagnosticar en que nivel de madurez se encuentra en la implementación, al evaluarse cada año a nivel de gerencia se puede definir el rumbo de los siguientes pasos, este diagnóstico consta de al menos tres niveles, y describe comportamientos esperados para pasar al siguiente nivel y planear próximos 5 años.

El diagnóstico consta al menos de tres niveles donde 1 es el nivel más básico donde sólo se usa la iniciativa en algunas áreas como HERRAMIENTAS, 3 se considera un SISTEMA, pero requiere esfuerzo de consultor para que se siga, 5 es un PRINCIPIO la gente lo usa en la vida diaria (Tabla 2).

Este diagnóstico hay que desarrollarlo para cada empresa y cada metodología que se quiera implementar y es recomendable hacerla con ayuda de un consultor experto y validada posteriormente por el comité para que quede adecuado al lenguaje y visión de la empresa. Es indispensable establecer mecanismos para determinar las prioridades en cada área de oportunidad, conjugando los objetivos de la empresa y los recursos disponibles para aprovechar dichas áreas.

En la investigación se encontraron 3 ejemplos: Hirano (2008), Productivity (2009) y el premio de Shingoprize (ver www.shingoprize.com).

\section{Cortez et al.}


Tabla 2. Modelo de Visión para evaluar Nivel de Madurez de iniciativas, Productivity (2009)

\begin{tabular}{|c|c|c|c|c|c|}
\hline \multicolumn{2}{|c|}{$\begin{array}{l}\text { CRITE- } \\
\text { RIO }\end{array}$} & $\begin{array}{l}\text { METODOS } \\
\text { CONFIABLES }\end{array}$ & $\begin{array}{l}\text { GRADO DE } \\
\text { DIFUSIÓN }\end{array}$ & $\begin{array}{c}\text { GRADO DE } \\
\text { INTEGRACION } \\
\text { INTERFUNCIONAL }\end{array}$ & RESULTADOS \\
\hline \multirow[b]{2}{*}{$\sum_{\bar{z}}^{\bar{z}}$} & \multirow{2}{*}{ 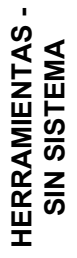 } & No hay sistema & $\begin{array}{l}\text { No se conoce, no } \\
\text { hay entrenamiento }\end{array}$ & Sólo por casualidad & $\begin{array}{l}\text { Muy por debajo } \\
\text { de la industria }\end{array}$ \\
\hline & & $\begin{array}{l}\text { Pobres } \\
\text { estándares }\end{array}$ & $\begin{array}{l}\text { Algunas personas } \\
\text { ocasionalmente lo } \\
\text { usan }\end{array}$ & $\begin{array}{l}\text { Fallas de } \\
\text { comunicación }\end{array}$ & $\begin{array}{l}\text { Resultado son } \\
\text { casualidad, no } \\
\text { hay medición }\end{array}$ \\
\hline \multirow{2}{*}{$\stackrel{N}{\sum}$} & \multirow{2}{*}{ 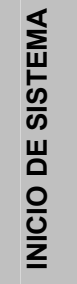 } & Sistema nuevo & $\begin{array}{l}\text { Entrenamiento } \\
\text { inicial }\end{array}$ & $\begin{array}{l}\text { Conceptualizado } \\
\text { pero no se } \\
\text { práctica }\end{array}$ & $\begin{array}{l}\text { Debajo de la } \\
\text { industria }\end{array}$ \\
\hline & & $\begin{array}{l}\text { Alguna } \\
\text { estandarización }\end{array}$ & $\begin{array}{l}\text { Proyectos piloto } \\
\text { en grandes áreas }\end{array}$ & $\begin{array}{l}\text { Fallas de } \\
\text { comunicación } \\
\text { detectadas }\end{array}$ & $\begin{array}{l}\text { Establece } \\
\text { objetivos } \\
\text { métricos } \\
\text { Tendencia } \\
\text { positivas }\end{array}$ \\
\hline \multirow{2}{*}{$\sum^{m}$} & \multirow{2}{*}{$\frac{\sum_{W}^{\frac{\Phi}{5}}}{\frac{\omega}{\omega}}$} & $\begin{array}{l}\text { Sistema } \\
\text { probado }\end{array}$ & Difusión horizontal & $\begin{array}{l}\text { Se practica en } \\
\text { algunos casos }\end{array}$ & $\begin{array}{l}\text { A la par con la } \\
\text { industria }\end{array}$ \\
\hline & & $\begin{array}{l}\text { Buena } \\
\text { estandarización }\end{array}$ & $\begin{array}{l}\text { Mayoría de áreas } \\
\text { principales, algunas } \\
\text { de apoyo }\end{array}$ & $\begin{array}{l}\text { Se hacen esfuerzos } \\
\text { para mejorar la } \\
\text { comunicación }\end{array}$ & $\begin{array}{l}\text { Tendencias } \\
\text { positivas en la } \\
\text { mayoría de las } \\
\text { áreas principales }\end{array}$ \\
\hline \multirow{2}{*}{$\sum_{z}^{+}$} & \multirow{2}{*}{ 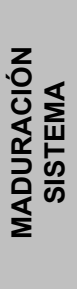 } & $\begin{array}{l}\text { Sistema con } \\
\text { ajustes }\end{array}$ & $\begin{array}{l}\text { Fuerte } \\
\text { implementa-ción }\end{array}$ & Buena integración & $\begin{array}{l}\text { Arriba el } \\
\text { promedio de la } \\
\text { industria }\end{array}$ \\
\hline & & $\begin{array}{l}\text { Excelente } \\
\text { estandarización }\end{array}$ & $\begin{array}{l}\text { Todas las áreas } \\
\text { principales y } \\
\text { muchas de apoyo }\end{array}$ & $\begin{array}{l}\text { Pocos de casos } \\
\text { de comunicación }\end{array}$ & $\begin{array}{l}\text { De bueno a } \\
\text { excelente en } \\
\text { áreas } \\
\text { principales }\end{array}$ \\
\hline \multirow{2}{*}{$\sum^{n}$} & \multirow{2}{*}{ 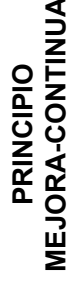 } & $\begin{array}{l}\text { Sistema } \\
\text { refinado }\end{array}$ & $\begin{array}{l}\text { Implementación } \\
\text { total }\end{array}$ & $\begin{array}{l}\text { Excelente } \\
\text { integración }\end{array}$ & Clase mundial \\
\hline & & $\begin{array}{l}\text { Mejoras } \\
\text { continuas al } \\
\text { sistema }\end{array}$ & $\begin{array}{l}\text { Todas las áreas } \\
\text { principales y de } \\
\text { apoyo }\end{array}$ & $\begin{array}{l}\text { Las fallas de } \\
\text { comunicación se } \\
\text { han eliminado. }\end{array}$ & $\begin{array}{l}\text { De bueno a } \\
\text { excelente en } \\
\text { áreas de apoyo }\end{array}$ \\
\hline
\end{tabular}

Manufactura Esbelta 


\section{Método empleado}

El estudio se lleva a cabo en un primer momento como una investigación documental cuyo propósito es identificar y asimilar conocimiento teórico y científico relevante que existe sobre los pasos para la implementación del Sistema de Gestión TPS (Tabla 1).

Para desarrollar esta investigación se buscó una empresa con varios años de experiencia en la implementación de diferentes iniciativas de Manufactura Esbelta. Para lo anterior, se seleccionó una planta de Acero Forjado ubicada en Santa Catarina N.L., en la que laboran 700 personas y que cuenta con 6 gerencias con más de 3 años de trabajo en la implementación de las iniciativas que a continuación se mencionan: 1) Eventos Kaizen, 2) Proyectos de Mejora DMAIC, 3) Equipos participativos, 4) Centro de entrenamiento, 5) Desarrollo de productos "Stage Gate", 6) TPM, 7) Estandarización, 8) 5'S, 9) Investigación y Desarrollo, y 10) Sistema de Ideas. Cabe señalar que para cada una de las iniciativas existe un consultor interno responsable de la implementación.

En la siguiente etapa del estudio se aplicó una encuesta tipo Likert a gerentes y consultores internos para la evaluación del diagnóstico del nivel de madurez de cada iniciativa adaptada de la Tabla 2 para las 6 gerencias de la empresa que aplican las 10 iniciativas. Los resultados se presentan en la Tabla 3. El nivel de madurez por gerencia representa la variable de salida. La escala de los niveles de madurez es del 1 al 5 , donde 1 indica que no existe sistema, 3 existe sistema y 5 implica la existencia de una mejora continua (Tabla 2).

Posteriormente, con base en los trabajos de Robert (2005) y Womack (1991) se definieron 19 variables independientes (X's) y se les asignó una unidad de medida (Tabla 4). Con lo anterior se desarrolló un cuestionario aplicándose un total de 60 encuestas correspondientes a los 10 consultores internos de cada iniciativa de las 6 gerencias.

\section{Cortez et al.}


Tabla 3. Nivel de madurez de las iniciativas de mejora por gerencia

\begin{tabular}{|c|c|c|c|c|c|c|c|}
\hline Enfoque & Iniciativa & $\begin{array}{l}\bar{\sigma} \\
\frac{\pi}{0} \\
\bar{\Phi} \\
\bar{d}\end{array}$ & 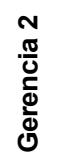 & 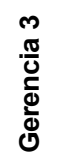 & 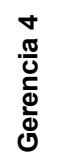 & $\begin{array}{l}n \\
\frac{\pi}{0} \\
\frac{\pi}{\omega} \\
\frac{\Phi}{\pi} \\
0\end{array}$ & $\begin{array}{l}0 \\
\frac{\pi}{0} \\
\frac{\pi}{0} \\
\frac{d}{20} \\
0\end{array}$ \\
\hline \multirow{2}{*}{$\begin{array}{c}\text { Solucion a } \\
\text { problemas corto } \\
\text { plazo }\end{array}$} & Eventos Kaizen & 2.75 & 2.52 & 2.96 & 1.80 & 2.20 & 2.85 \\
\hline & Proyectos Mejora & 3.13 & 3.22 & 1.80 & 1.73 & 1.80 & 3.30 \\
\hline \multirow{5}{*}{$\begin{array}{l}\text { Sostenimiento a } \\
\text { largo plazo }\end{array}$} & Equipos Participativos & 2.37 & 2.29 & 2.31 & 2.00 & 1.80 & 1.80 \\
\hline & Centro de entrenamiento & 2.47 & 2.65 & 3.18 & 2.11 & 1.65 & 2.00 \\
\hline & $\underline{5 S^{\prime} \mathrm{s}}$ & 2.72 & 2.30 & 2.47 & 2.47 & 1.82 & 1.87 \\
\hline & $\frac{\text { TPM (Mantenimiento }}{\text { Productivo Total) }}$ & 1.66 & 1.66 & 1.28 & 1.28 & 1.84 & 1.20 \\
\hline & Estandarización E1 & 2.50 & 2.68 & 2.17 & 2.63 & 2.20 & 2.25 \\
\hline \multirow{4}{*}{ Innovación } & $\frac{\text { Desarrollo de Productos }}{\text { (Stage Gate) }}$ & 0.00 & 0.00 & 0.00 & 0.00 & 0.00 & 0.00 \\
\hline & Investigación y Desarrollo & 1.50 & 1.50 & 1.00 & 1.00 & 0.00 & 1.50 \\
\hline & $\frac{\text { Sistema de Ideas y }}{\text { sugerencias }}$ & 0.50 & 1.00 & 0.50 & 0.50 & 1.00 & 0.50 \\
\hline & $\begin{array}{l}\text { Promedio de la iniciativa por } \\
\text { gerencia }\end{array}$ & 2.51 & 2.47 & 2.31 & 2.00 & 1.90 & 2.18 \\
\hline
\end{tabular}

Tabla 4. Pasos de implementación y factores

\begin{tabular}{|c|c|c|}
\hline$\frac{\frac{0}{0}}{\frac{0}{\frac{\pi}{2}}}$ & $\begin{array}{l}\text { Iniciativas Empresa } \\
\text { vs Pasos para } \\
\text { implementación }\end{array}$ & Unidad de medida \\
\hline Y & $\begin{array}{l}\text { Y1: Nivel de madurez } \\
\text { de la iniciativa }\end{array}$ & 1 Herramientas, 3 Sistemas y 5 Principios \\
\hline X1: & Años en la empresa & Número de años hasta Dic 2009 \\
\hline $\mathrm{X} 2$ : & $\begin{array}{l}\text { Venta de idea a la } \\
\text { Dirección del cambio }\end{array}$ & $\begin{array}{l}\text { (1) Recomendación Corporativo (2) Recomendación experiencias } \\
\text { de otras plantas-consultores, (3) Solicitada por cliente (4) } \\
\text { Ejecutivos de la empresa con experiencia anterior de manufactura } \\
\text { esbelta. (5) Se expuso y no se vendió idea) }\end{array}$ \\
\hline X3: & $\begin{array}{l}\text { Objetivo principal al } \\
\text { arrancar la iniciativa }\end{array}$ & $\begin{array}{l}\text { (1) Mejorar procesos actuales Corto plazo (2) Estrategia de } \\
\text { supervivencia, (perdida de mercado x competidor, precio bajar } \\
\text { costos, tiempo de entrega, mejora calidad), (3) Busca crear Cultura } \\
\text { Mejora y Desarrollo de personas (4) Estrategia de crecimiento } \\
\text { Largo Plazo (5) Estrategia de sustentabilidad proceso Largo Plazo) }\end{array}$ \\
\hline X4: & Prioridad Estratégica & $\begin{array}{l}\text { (1) Si se incluyo como prioridad de implementar en planeación } \\
\text { estratégica (2) no se incluyo }\end{array}$ \\
\hline X5: & Agente de Cambio inicial & $\begin{array}{l}\text { (1) Consultor externo-Líder tiempo parcial, (2) Consultor externo - } \\
\text { Consultor Interno } \\
\text { (3) } 1 \text { Consultor Interno }\end{array}$ \\
\hline
\end{tabular}

\section{Manufactura Esbelta}




\begin{tabular}{|c|c|c|}
\hline 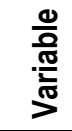 & $\begin{array}{l}\text { Iniciativas Empresa } \\
\text { vs Pasos para } \\
\text { implementación }\end{array}$ & Unidad de medida \\
\hline $\mathrm{X} 6$ : & $\begin{array}{l}\text { Frecuencia reunión } \\
\text { comité con Dirección } \\
\text { (1er año) }\end{array}$ & $\begin{array}{l}\text { (1) No se creo comité (2) Dos/tres veces x mes (3)1 vez por mes } \\
\text { (4) Cada } 3 \text { meses. }\end{array}$ \\
\hline $\mathrm{X} 7:$ & $\begin{array}{l}\text { Frecuencia de } \\
\text { seguimiento con } \\
\text { Gerentes o Jefes }\end{array}$ & $\begin{array}{l}\text { (1) No se creo comité (2) } 1.5 \text { a } 3 \text { veces x mes (3)1 vez por mes ( } 4 \text { ) } \\
\text { Cada } 3 \text { meses. }\end{array}$ \\
\hline X8: & $\begin{array}{l}\text { Gente que participa } x \\
\text { mes }\end{array}$ & Cantidad de personas participan $\mathrm{x}$ mes $(0)$ No arranco \\
\hline X9: & $\begin{array}{l}\text { Adaptación a la } \\
\text { metodología }\end{array}$ & $\begin{array}{l}\text { Poner del } 1 \text { al } 5 \text { (1) Copia exacta del consultor (5) Totalmente } \\
\text { nueva salida de FRISA (0) No arrancó }\end{array}$ \\
\hline X10: & $\begin{array}{l}\% \text { involucramiento del } \\
\text { director-Gerente }\end{array}$ & (1) Bajo (3) Medio (5) Alto (0) No se arrancó \\
\hline X11: & Definir Visión & $\begin{array}{l}\text { Cuenta con visión y objetivos a= (1) } 6 \text { meses, (2) se tiene idea de } \\
\text { lo que hay que hacer y donde llegar en } 5 \text { años pero no esta escrito } \\
\text { al detalle (3) Se cuenta con una visión a } 5 \text { años escrita al detalle } \\
\text { con elementos. (0) No arrancó }\end{array}$ \\
\hline X12: & $\begin{array}{l}\text { Establecer Métricos y } \\
\text { metas }\end{array}$ & $\begin{array}{l}\text { El resultado esperado tiene métricos para el seguimiento (1) No } \\
\text { existen (2) No siempre adecuados (3) Existen los adecuados (0) } \\
\text { No arrancó }\end{array}$ \\
\hline X13: & $\begin{array}{l}10 \text { Elaborar Plan de } \\
\text { Mejora }\end{array}$ & 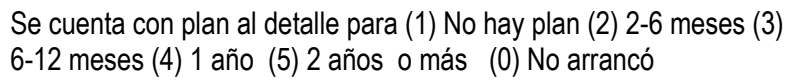 \\
\hline X14: & $\begin{array}{l}12 \text { Vencer la Resistencia } \\
\text { al Cambio }\end{array}$ & $\begin{array}{l}\text { (1) No se da tiempo la gente, (2) No convencidos gerentes (3) No } \\
\text { convencidos mandos medios (4) Consultor interno no hábil para } \\
\text { liderar con obstáculos (5) Falta de recursos por no ser prioridad (6) } \\
\text { Falta entrenamiento }\end{array}$ \\
\hline X15: & $\begin{array}{l}\text { Implementar plan por } \\
\text { consultor-líder de la } \\
\text { iniciativa }\end{array}$ & $\begin{array}{l}\text { (1) Consultor convence a los miembros de la gerencia (1) Alto } \\
100 / 90 \% \text {, (2) medio } 90 / 60 \% \text { (3) bajo abajo } 60 \%\end{array}$ \\
\hline X16: & $\begin{array}{l}\text { Comprobar los } \\
\text { Resultados }\end{array}$ & $\begin{array}{l}\text { (1) Cumplieron en eficiencia y eficacia lo esperado, (2) Cumplieron } \\
\text { meta solo algunas áreas (3) No cumplieron meta } \\
\text { (0) No arrancó }\end{array}$ \\
\hline X17: & Estandarizar las mejoras & $\begin{array}{l}\text { (1) Se aplico mejoras en otras áreas y se volvió ajustar visión (2) } \\
\text { No se replico ni ajusto visión con cambios } \\
\text { (0) No arrancó }\end{array}$ \\
\hline X18: & Reconocimiento & $\begin{array}{l}\text { (1) No hay (2)Reconocimiento en revista y juntas (3) Revistas, } \\
\text { Juntas, foro anual, (4) económico, competencia fuera de Frisa. (0) } \\
\text { No arrancó }\end{array}$ \\
\hline X19: & Valores y cultura & $\begin{array}{l}\text { (1) No está declarado en los valores ni se promueve (2) Está } \\
\text { implícito en los valores y se promueve (3) Esta claramente escrito } \\
\text { en los valores y se promueve (0) No arrancó }\end{array}$ \\
\hline
\end{tabular}

\section{Cortez et al.}




\section{Resultados estadísticos de la muestra}

Para obtener el peso de cada uno de los 19 pasos en la implementación y dada la naturaleza de la investigación se selección un método estadístico de análisis multivariante, esto es, la metodología del análisis regresión lineal múltiple empleando el software Minitab. La ecuación resultante se presenta a continuación (el término de error de la regresión se representa con la variable $\varepsilon$ ):

$$
\begin{aligned}
& Y=100-0.877 X_{1}-7.81 X_{2}+216 X_{3}-122 X_{4}-321 X_{5}-15.2 X_{6}+ \\
& 0.222 X_{7}+0.000317 X_{8}+120 X_{9}+0.343 X_{10}+3.3022 X_{11}+0.312 X_{12}+ \\
& 0.525 X_{13}+0.0104 X_{14}+0.359 X_{15}+2.3571 X_{16}-9.292 X_{17}+0.0112 X_{18}- \\
& 0.5521 X_{19}+\varepsilon
\end{aligned}
$$

Cabe señalar que se obtuvo una $\mathrm{R}^{2}$ ajustada de $99 \%$ y que las variables $X_{8}, X_{14}, X_{15}, X_{18}$, no son significativas en el modelo, por tener un $p$ value $>.05$. Por ello, optamos por estimar otro modelo eliminando estas variables. La $\mathrm{X}_{2}$ (Venta de idea), $\mathrm{X}_{5}$ (Agente de cambio), $\mathrm{X}_{6}$ (Frecuencia reunión dirección), $X_{10}$ (\%involucramiento gerencia), $X_{11}$ (Visión), $X_{12}$ (Métricos y metas), $X_{13}$ (Plan), $X_{19}$ (Cultura), tienen mayor impacto en la $Y$. El modelo simplificado queda de la siguiente forma:

$$
\begin{aligned}
& Y=224+0.757 X_{2}-0.152 X_{3}-3.31 X_{4}+5.57 X_{5}-4.30 X_{6} \\
& +0.102 X_{7}-0.288 X_{9}+0.178 X_{10}+3.30 X_{11}+0.313 X_{12} \\
& +0.284 X_{13}+236 X_{16}-9.29 X_{17}-0.552 X_{19}+\varepsilon
\end{aligned}
$$

Todas las betas de las variables son estadísticamente sigificativas, siendo $X_{17}, X_{5}, X_{6}$, las que mayor impacto tienen en la variable de madurez. Además, el $95 \%$ de la variabilidad de la madurez (variable dependiente) se debe a las variables independientes del modelo y más en estas 8 variables: $X_{2}, X_{4}, X_{5}, X_{6}, X_{11}, X_{16}, X_{17}, X_{19}$

\section{Manufactura Esbelta}




\section{Ciclo de vida de las iniciativas}

Buscando traducir el concepto abstracto de "Cultura Lean" en algo concreto que alinee a los comportamientos y principios esperados de las personas, a prácticas y herramientas que monitoreen la implementación en las diferentes etapas de implementación, identifiquen fallas y crisis, y ayude a elevar el nivel de madurez de la implementación, se encontró en la Ciencia de la Psicología, el desarrollo psicosocial de Erik Erikson.

La investigación de Erik Erison presenta el diagrama del adulto de acuerdo a VII estadios del ciclo completo de vida. Cada estadio menciona las crisis por las que pasa la persona, las relaciones sociales significativas, fuerzas básicas para superar dichas crisis y las fuerzas distónicas, también los principios y los sistemas sociales relacionados (Bordignon 2005).

Buscando una adaptación de los ciclos de vida de Erikson a los ciclos de vida de la implementación se construyó la siguiente tabla donde se relacionan los estadios, con las variables significativas en la empresa estudiada y además que tenían mayor impacto $\left(X_{2}, X_{4}, X_{5}, X_{6}, X_{11}, X_{16}, X_{17}\right.$, $\left.X_{19}\right)$.

El modelo propuesto en este artículo, presenta un ciclo de cambio con fundamento en el principio organísmico de la epigenética en un intento de encontrar respuestas para lograr el éxito y permanencia de este Sistema de Producción, detectar las crisis por las que pasa cada estadío y cómo los principios de la manufactura esbelta sugieren soluciones para evitar los problemas, con los resultados de la misma (Tabla 3).

Para interpretar la tabla se debe leer por columna, por ejemplo, el estadío de la concepción se relaciona con $\mathrm{X}_{2}$ (venta de idea de la dirección), la crisis por la que se pasaría sería (Confianza vs Desconfianza) la confianza es la esperanza de irnos mejor a largo plazo y desconfiana el miedo de que no funcione.

El pensamiento estratégico es operar bajo un modelo de producción flexible 0 en masa y así sucecivamente hacia acada una de las filas hasta llegar al principio de ME que no muestra las fuerzas integradoras que apoyan al principio de ME y los rituales desintegradores que irían en contra del mismo.

\section{Cortez et al.}


Tabla 6. Ciclo de vida de la implementación ME

\begin{tabular}{|c|c|c|c|c|c|c|c|c|}
\hline 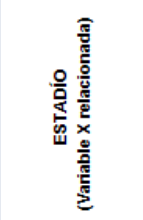 & 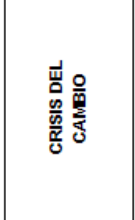 & 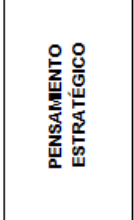 & 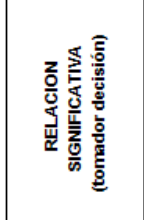 & 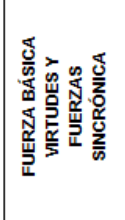 & 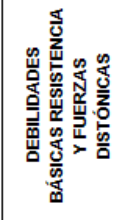 & 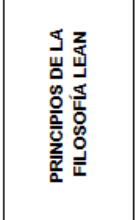 & 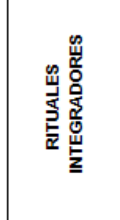 & 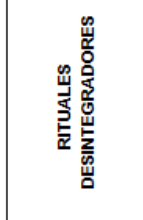 \\
\hline $\begin{array}{l}\text { CONCEPCION } \\
\text { (X2 Vender idea a } \\
\text { dirección X5 } \\
\text { Agente de cambio } \\
\text { inicial) }\end{array}$ & \begin{tabular}{|c|} 
CONFIANZA Y \\
FE VS. \\
DESCONFIANZ \\
A (personas) \\
\end{tabular} & $\begin{array}{l}\text { PRODUCCIÓN } \\
\text { FLEXIBLE VS. } \\
\text { PRODUCCIÓN } \\
\text { EN MASA }\end{array}$ & $\begin{array}{l}\text { CONSULTOR } \\
\text { INTERNO- } \\
\text { EXTERNO }\end{array}$ & $\begin{array}{l}\text { ESPERANZA } \\
\text { Propósito } \\
\text { común que } \\
\text { supera el } \\
\text { ganar dinero }\end{array}$ & \begin{tabular}{|l} 
DESCONFIANZA \\
1. Negación y \\
minimización \\
2 Miedo \\
incertidumbre al \\
cambio
\end{tabular} & \begin{tabular}{|c|} 
TOME \\
DECISIONES \\
CON VISION A \\
LARGO PLAZO \\
AUN \\
EXPENSAS DE \\
RESULTADDOS \\
CORTO PLAZO \\
\end{tabular} & \begin{tabular}{|l|} 
LIDERAZGO \\
VISIONARIO \\
\end{tabular} & $\begin{array}{c}\text { IDOLATRIA } \\
\text { Sólo enfoque a } \\
\text { ahorrar, resolver } \\
\text { problemas de } \\
\text { calidad y de } \\
\text { servicio corto } \\
\text { plazo }\end{array}$ \\
\hline $\begin{array}{c}\text { BEBE } \\
\text { X5 Agente de } \\
\text { cambio inicial, X6 } \\
\text { (seguimiento } \\
\text { comité dirección) }\end{array}$ & \begin{tabular}{|c|} 
MADUREZ VS. \\
INMADUREZ
\end{tabular} & \begin{tabular}{|c|} 
DE PERSONAS \\
EN PUESTOS \\
VS. PERSONAS \\
LIDERES DE \\
CAMBIO
\end{tabular} & \begin{tabular}{|} 
CONSULTOR \\
INTERNO- \\
EXTERNO, \\
DIRECTOR, \\
GERENTES \\
(EQUIPO GUIA)
\end{tabular} & \begin{tabular}{|c|} 
MADUREZ \\
Estamos \\
entrenados, \\
requerimos \\
guia consultor \\
y voluntad \\
para cambio
\end{tabular} & $\begin{array}{l}\text { INMADUREZ } \\
\text { Resistencia } \\
\text { cambio, } \\
\text { inseguridad, } \\
\text { factores } \\
\text { economicos }\end{array}$ & \begin{tabular}{c|} 
DESARROLLE \\
PERSONASY \\
EQUIPOS QUE \\
SIGAN LA \\
FILOSOFIA.
\end{tabular} & \begin{tabular}{|l} 
CRECIMIIENTO \\
LíDERES \\
INTERNOS, \\
Cree cultura \\
fuerte en \\
valores y \\
creencias
\end{tabular} & $\begin{array}{c}\text { MORALISMO } \\
\text { Capacitación con } \\
\text { un enfoque } \\
\text { limitado al cambio } \\
\end{array}$ \\
\hline $\begin{array}{l}\text { INFANCIA } \\
\text { (X11 Definir } \\
\text { Visión) }\end{array}$ & \begin{tabular}{|c} 
VISIÓN \\
HOLISTICA VS. \\
VISIÓN \\
INDNIDUAL
\end{tabular} & $\begin{array}{c}\text { Procesos de } \\
\text { producción en } \\
\text { BATCH contra } \\
\text { flujo continuo } \\
\text { de alto valor } \\
\text { añadido }\end{array}$ & \begin{tabular}{|c|} 
CONSULTORY \\
MANDOS \\
MEDIOS \\
(JEFESY \\
SUPERVISORES)
\end{tabular} & $\begin{array}{c}\text { VISIÓN } \\
\text { HOLISTICA de } \\
\text { sistema, } \\
\text { reducción del } \\
\text { tiempo total de } \\
\text { ciclo y } \\
\text { desperdicios }\end{array}$ & $\begin{array}{l}\text { VISIÓN } \\
\text { INDNIDUAL } \\
\text { del trabajo } \\
\text { Hábitos de } \\
\text { trabajo } \\
\text { anteriores }\end{array}$ & \begin{tabular}{|l|} 
CREE UN FLUJO \\
CONTINUO \\
PARA HACER \\
QUE LOS \\
PROBLEMAS \\
SALGAN. \\
\end{tabular} & $\begin{array}{c}\text { ESTANDARI- } \\
\text { ZACIÓN Y } \\
\text { SEGUIMIENTO } \\
\end{array}$ & $\begin{array}{c}\text { FORIMALISMO } \\
\text { Sobrecargar al } \\
\text { personal y a los } \\
\text { equipos }\end{array}$ \\
\hline $\begin{array}{l}\text { ADOLESCENCIA } \\
\text { (X10 } \\
\text { Involucramiento } \\
\text { de los gerentes) }\end{array}$ & \begin{tabular}{|c|} 
INVOLUCRA- \\
MENTO VS. NO \\
INVOLUCRA- \\
MENTO
\end{tabular} & \begin{tabular}{|c|} 
Integrar \\
iniciativas de \\
mejora entre las \\
10 primeras de \\
Planeación \\
Estratégica y \\
en la \\
declaración de \\
valores \\
\end{tabular} & \begin{tabular}{|c|} 
CONSULTOR \\
INTERNO O \\
EXTERNO \\
YLIDERES \\
FORIMALES E \\
INFORMALES DE \\
CAMBBIO \\
\end{tabular} & $\begin{array}{l}\text { INVOLUCRA- } \\
\text { MEENTO } \\
\text { Enseñar a } \\
\text { individuos a } \\
\text { trabajar como } \\
\text { equipo para } \\
\text { logar objetivos }\end{array}$ & $\begin{array}{l}\text { NO INVOLUCRA. } \\
\text { MENTO } \\
\text { Las mejoras y } \\
\text { uso de } \\
\text { herramientas } \\
\text { dependen de los } \\
\text { consultores }\end{array}$ & \begin{tabular}{|c|} 
DESARROLLE \\
PERSONASY \\
EQUIPOS \\
EXCEPCIONALE \\
S QUE SIGAN \\
LA FILOSOFIA \\
DE LA \\
EMPRESA \\
\end{tabular} & \begin{tabular}{|c|} 
CREAR \\
CULTURA \\
valores y \\
creencias \\
duren años. \\
AUTONOMIA \\
uso de \\
herramientas \\
en su rutina \\
diaria. \\
\end{tabular} & $\begin{array}{c}\text { AUTORTARISMO } \\
\text { (Poder } \\
\text { degenerado) } \\
\text { TOTALTARISMO } \\
\text { (Fanatismo) } \\
\\
\end{array}$ \\
\hline $\begin{array}{c}\text { ADULTO } \\
\text { (X12 Establecer } \\
\text { métricos y metas } \\
\text { X13 Plan de } \\
\text { mejora) }\end{array}$ & \begin{tabular}{|c|} 
BUROCRA- \\
CIA VRS \\
PRAGMATISMO \\
\end{tabular} & \begin{tabular}{|c|} 
Metodologias y \\
estructura \\
soporte esbelta
\end{tabular} & $\begin{array}{c}\text { DIRECTOR, } \\
\text { GERENTES, } \\
\text { CONSULTOR } \\
\text { INTERNO }\end{array}$ & $\begin{array}{l}\text { BUROCRACIA } \\
\text { Metodología } \\
\text { como fin y no } \\
\text { como medio, } \\
\text { pasos o } \\
\text { documentación } \\
\text { innecesaria } \\
\end{array}$ & $\begin{array}{c}\text { PRAGMATISMO } \\
\text { Mantener simple } \\
\text { y práctico las } \\
\text { iniciativas } \\
\\
\end{array}$ & \begin{tabular}{|c|} 
VAYAA A \\
VERLO POR SI \\
MISMO PARA \\
COMPRENDER \\
A FONDO LA \\
STUACIÓN. \\
\end{tabular} & \begin{tabular}{|c|} 
Dirección y \\
ejecutivos \\
deben ir a la \\
fuente, en \\
lugar de \\
teorizar en \\
salas o PC.
\end{tabular} & $\begin{array}{l}\text { FORMALISMO, } \\
\text { RTUALISMOS }\end{array}$ \\
\hline $\begin{array}{l}\text { MADUREZ } \\
\text { (X19 valores y } \\
\text { cultura) }\end{array}$ & $\begin{array}{c}\text { MORAL } \\
\text { HONESTIDAD } \\
\text { VS. } \\
\text { DESHONESTO }\end{array}$ & $\begin{array}{l}\text { Desarrollo de } \\
\text { proveedores y } \\
\text { socios }\end{array}$ & \begin{tabular}{|c|} 
DIRECTOR \\
Gerente de \\
abastecimientos
\end{tabular} & $\begin{array}{c}\text { HONESTIDAD } \\
\text { Cumplir } \\
\text { especificacion } \\
\text { es de Calidad } \\
\text { Costo y } \\
\text { servicio }\end{array}$ & \begin{tabular}{|l} 
DESHONESTO \\
Cumplir \\
compromisos. \\
Mandar \\
materiales con \\
problemas
\end{tabular} & \begin{tabular}{|l|} 
RESPETE RED \\
DE SOCIO Y \\
PROVEEDORES \\
DESAFLÁNDO Y \\
AYUDÁNDOLO \\
S A MEJORAR. \\
\end{tabular} & \begin{tabular}{|c|} 
Tráte \\
proveedores \\
como una \\
extensión de \\
su empresa, \\
muestre \\
valores, pida \\
objetivos \\
\end{tabular} & MORALISMO \\
\hline $\begin{array}{l}\text { MADUREZ O } \\
\text { VEJJEZ } \\
\text { (X19 Cultura) }\end{array}$ & $\begin{array}{l}\text { APRENDIZAJE } \\
\text { Y MEJJRA } \\
\text { CONTINUA VS. } \\
\text { CAMBIO } \\
\text { DIRECTRIZ }\end{array}$ & $\begin{array}{c}\text { Venta de la } \\
\text { compañia, } \\
\text { cambio de } \\
\text { director de } \\
\text { planta }\end{array}$ & $\begin{array}{c}\text { Dueño, } \\
\text { Corporativo, } \\
\text { Director de } \\
\text { Planta }\end{array}$ & $\begin{array}{l}\text { APRENDIZAJE } \\
\text { Y MEJORA } \\
\text { CONTINUA } \\
\text { Proteja } \\
\text { conocimiento, } \\
\text { promociones } \\
\text { lentas y } \\
\text { sistemas de } \\
\text { sucesión. }\end{array}$ & $\begin{array}{l}\text { CAMBIO } \\
\text { DIRECTRIZ } \\
\text { Cambio de } \\
\text { director, con } \\
\text { una forma } \\
\text { diferente de } \\
\text { operar y } \\
\text { administrar a } \\
\text { Lean }\end{array}$ & \begin{tabular}{|c|} 
APRENDE \\
MEDIANTE LA \\
REFLEXIÓN \\
CONSTANTE \\
(HANSEI) Y LA \\
MEJORA \\
CONTINUA \\
(KAIZEN) \\
\end{tabular} & \begin{tabular}{|c|} 
Estandarizar \\
mejores \\
prácticas y \\
difunda, en \\
lugar de \\
reinventar \\
con todo \\
nuevo \\
proyecto o \\
director \\
\end{tabular} & $\begin{array}{c}\text { DOGMATISMO } \\
\text { (Ritualismo) }\end{array}$ \\
\hline
\end{tabular}

Manufactura Esbelta 


\section{Conclusión y Recomendaciones}

El concepto de ME ha evolucionado desde los cuarentas existen varias propuestas de implementación destacamos los trabajos de Robert (2005) y Womack (1991). Es importante dejar enfatizar la visón año por año para medir el avance en el nivel de madurez (variable dependiente).

Se acepta la hipótesis nula: el nivel de madurez de las iniciativas está relacionado con los pasos propuestos de implementación. Los pasos de implementación que salieron significativos para esta empresa son debe a las variables independientes del modelo y más en estas 8 variables: $X_{2}$, (venta de idea de la dirección del cambio) $X_{4}$ (prioridad estratégica), $X_{5}$ (agente de cambio), $X_{6}$ (frecuencia de reunión con comité), $X_{11}$ (definir visión), $X_{16}$ (comprobar resultados), $X_{17}$ (estandarizar las mejoras), $X_{19}$ (valores y cultuta).

Se recomienda elaborar una visión y posterior diagnostico para establecer un punto de partida y el rumbo de los siguientes pasos para cada gerencia o empresa. Este diagnostico es recomendable hacerlo con un consultor externo en un inicio. Además se siguiere hacer un diagnostico una vez al año para medir el avance en la implementación de la iniciativa.

La tabla de Ciclos de vida expuesta anteriormente nos da algunas respuestas a ambas preguntas, al comparar los estadios, crisis y principios lean que ayudan a guiar y hacer que cada paso permanezca a través del tiempo, sin embargo dejo claro que requiere ampliar para futuras investigaciones la muestra y así tener un mayor número de casos de estudio para fundamentar la selección de la muestra y generalizar los resultados en diferentes sectores.

\section{Referencias}

Arrieta, J.G., V.E. Botero \& M.J. Romano, 2010. Benchmarking about lean manufacturing in the textile sector in Medellin, Journal of Economist and Administrative Science, 15(28): 141-154

Barney, M., 2002. Motorola second generation, ASQ org, Six Sigma Forum Magazine, 1(3):13-16.

Beer, M. \& N. Nohria, 2000. Cracking the code of change, Harvard Business Review, mayojunio: $15-25$

\section{Cortez et al.}


Brearley, M., 2000. Teams: Lessons from the World of Sport, BJM: British Medical Journal, 321 (7269): 1141-1143.

Bordignon, N.A., 2005. El desarrollo psicosocial de Erik Erikson. El diagrama epigenético del adulto, Revista Lasallista de Investigación, Colombia, 2(2): 50-63.

Garvin, D.A. \& M.A. Roberto, 2005. Change Through Persuasion, Harvard Business Review, febrero: 26-35

Goyal, S.K. \& S.G. Deshmukh, 1993. A critique of the literature on just-in-time manufacturing, International Journal of Operations \& Production Management, 12(1): 18-28.

Harry, M. \& D. Crawford, 2005. Six sigma: the next generation, Machine Design, 77(4): 126132.

Hiroyuki, H., 2008. JIT implementation manual: The complete guide to just-in-time manufacturing, 2a ed., Boca Raton, Fla: CRC Press.

Imai, M., 1991. Kaizen La clave de la ventaja competitiva japonesa, México: Compañia Editorial Continental.

Japan International Cooperation Agency, 2009. Modelo de implementación de herramientas japonesas de mejora, Diplomado para consultores PYME, México: Secretaría de Economía.

Kartha, C.P., 2004. A comparison of ISO 9000:2000 quality system standards, QS9000, ISO/TS 16949 and Baldrige criteria, The TQM Magazine, 16(5): 331-340.

Kim, W.C. \& R. Mauborge, 2003. Tipping Point Leadership, Harvard Business Review, abril: $37-47$.

Koeningsaecker G.K., 2009. Leading the lean enterprise transformation, New York: Productivity Press.

Kotter, J.P., 2007. Leading change: Why transformation efforts fail, Harvard Business Review, enero: 4-10.

Lean Enterprise, 2007. Encuesta Lean 2007.El Estado Lean www.lean.org.

Liker, J.L., 2006. Las claves del éxito de Toyota: 14 principios de gestión del fabricante más grande del mundo, Barcelona: Gestión 2000.

Mann, D., 2005. Creating a lean culture, Boca Raton: CRC Press.

Niebel, B., 2001. Ingeniería Industrial, México, D.F: Alfaomega.

Ohno, T., 1988. Toyota production system: beyond large-scale production, New York: Productivity Press.

Robert, A., 2005. Factores que facilitan el éxito y la continuidad de los equipos de mejora en las empresas industriales: Modelo de implantación, aplicación y medición de los resultados en una empresa piloto. Tesis doctoral. Universidad Politécnica de Cataluña.

Shingo Prize, 2010. Model \& application guidelines, Logan, Utah: Jon M. Huntsman School of Business.

Shah, R. \& P.T. Ward, 2003. Lean manufacturing: Context, practice bundles, and performance, Journal of operations management, 21(2): 129-149.

Shingo, S., 1987. Non-Stock Production: The Shingo System for Continuous Improvement. Cambridge, Mass: Productivity Press.

Socconini, L., 2009. Lean manufacturing, México, D.F: Ed Norma.

\section{Manufactura Esbelta}


Wendell, L.F. \& H.B. Jr. Cecil, 1996. Desarrollo Organizacional: Aportaciones de las ciencias de la conducta para el mejoramiento de la organización, México, D.F: Prentice-Hall.

White, R.E., 1999. JIT Manufacturing: A Survey of implementations in small and large U.S. manufacturers, Management Science:1-15.

Womack, J.P., D.T. Jones \& D. Roos, 1991. The machine that change the world: The story of lean production. New York: Harper Perennial.

Womack, J P. \& D.T. Jones, 1996. Lean Thinking: banish waste and create wealth in your corporation, New York: Simon \& Schuster.

Wong, Y.C., K.Y. Wong \& A. Ali, 2009. A Study on lean manufacturing implementation in the Malaysian electrical and electronics industry, European Journal of Scientific Research, 38(4): 521-535.

\section{Cortez et al.}

